Supporting Information

Maximizing contact of supersoft bottlebrush networks with rough surfaces to promote particulate removal

Teresa T. Duncan ${ }^{\dagger}$ Edwin P. Chan ${ }^{\dagger *}$, Kathryn L. Beers ${ }^{\dagger}$

${ }^{\dagger}$ Material Measurement Laboratory, National Institute of Standards and Technology,

Gaithersburg, Maryland 20899, United States

*Corresponding author

Email: edwin.chan@nist.gov 


\section{Characterization of BBNs}

Methods. Nuclear magnetic resonance (NMR) spectra were obtained with an Avance II $600 \mathrm{MHz}$ Bruker spectrometer. Gel permeation chromatography (GPC) data were acquired relative to polystyrene standards with a Waters instrument equipped with three mixed bed columns (HR0.5, HR3, and HR4) at a flow rate of $1 \mathrm{~mL} / \mathrm{min}$ and a constant temperature of $25^{\circ} \mathrm{C}$. A Thermo Fisher MARS III rheometer with a cone-plate geometry was used to collect strain and frequency sweeps of bottlebrush networks. Samples were pre-trimmed and placed on the rheometer base, and the tool was lowered enough to ensure good contact without causing the sample perimeter to bulge $\left(\mathrm{F}_{\mathrm{N}}\right.$ of $0.8 \mathrm{~Pa}$ for molar ratios of 0:1 and 10:1, 0.5 Pa for a molar ratio of 30:1, and $0.06 \mathrm{~Pa}$ for a molar ratio of 50:1 macromonomer (MM) to crosslinker (XL)). Strain sweeps were performed with an angular frequency of $6 \mathrm{rad} / \mathrm{s}$ and frequency sweeps were performed in the linear viscoelastic region. The equipment and instruments or materials are identified in the paper in order to adequately specify the experimental details. Such identification does not imply recommendation by National Institute of Standards and Technology, nor does it imply the materials are necessarily the best available for the purpose.

Table S1. $\mathrm{M}_{\mathrm{n}}$ of starting materials used to produce bottlebrush networks

\begin{tabular}{ccc}
\hline Starting Material & M $_{\mathbf{n}}$ from ${ }^{\mathbf{1}} \mathbf{H ~ N M R}^{\mathbf{a}}$ & $\mathbf{M}_{\mathbf{n}}$ from $_{\mathbf{~ G P C}}$ \\
\hline MCR-M11 & $980 \mathrm{~g} / \mathrm{mol}$ & $960 \mathrm{~g} / \mathrm{mol}$ \\
DMS-R22 & $8,800 \mathrm{~g} / \mathrm{mol}$ & $11,600 \mathrm{~g} / \mathrm{mol}$ \\
\hline
\end{tabular}
a. In $\mathrm{CDCl}_{3}$ at $25^{\circ} \mathrm{C}(600 \mathrm{MHz})$
b. In THF at $25^{\circ} \mathrm{C}$

Table S2. Maximum contact radius $\left(a_{\max }\right)$ and sample thickness $(t)$ during CAT experiments for select BBN materials

\begin{tabular}{ccccc}
\hline Molar ratio of MM to XL & $\boldsymbol{G}(\mathbf{k P a})$ & $\boldsymbol{a}_{\max }(\boldsymbol{\mu m})^{\mathbf{a}}$ & $\boldsymbol{t}(\boldsymbol{\mu m})$ & $\boldsymbol{a}$ \\
\hline $0: 1$ & 114 & $162 \pm 3$ & 3200 & 0.05 \\
$10: 1$ & 21 & $238 \pm 26$ & 3100 & 0.08 \\
$30: 1$ & 2 & $541 \pm 58$ & 2600 & 0.21 \\
$50: 1$ & 1 & $566 \pm 76$ & 2600 & 0.22 \\
\hline
\end{tabular}

a. Average value from three trials on one sample with associated standard deviation 


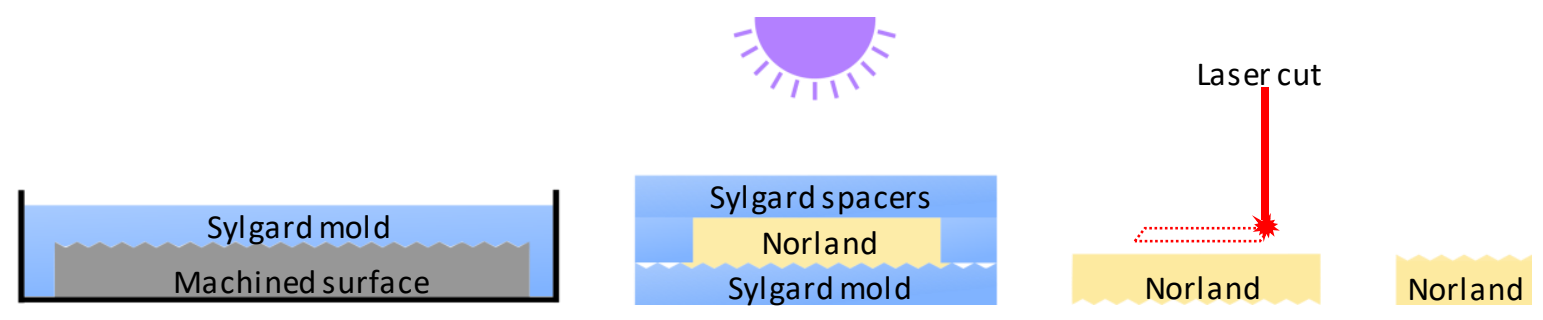

Figure S1. Schematic representing preparation of model rough surfaces.
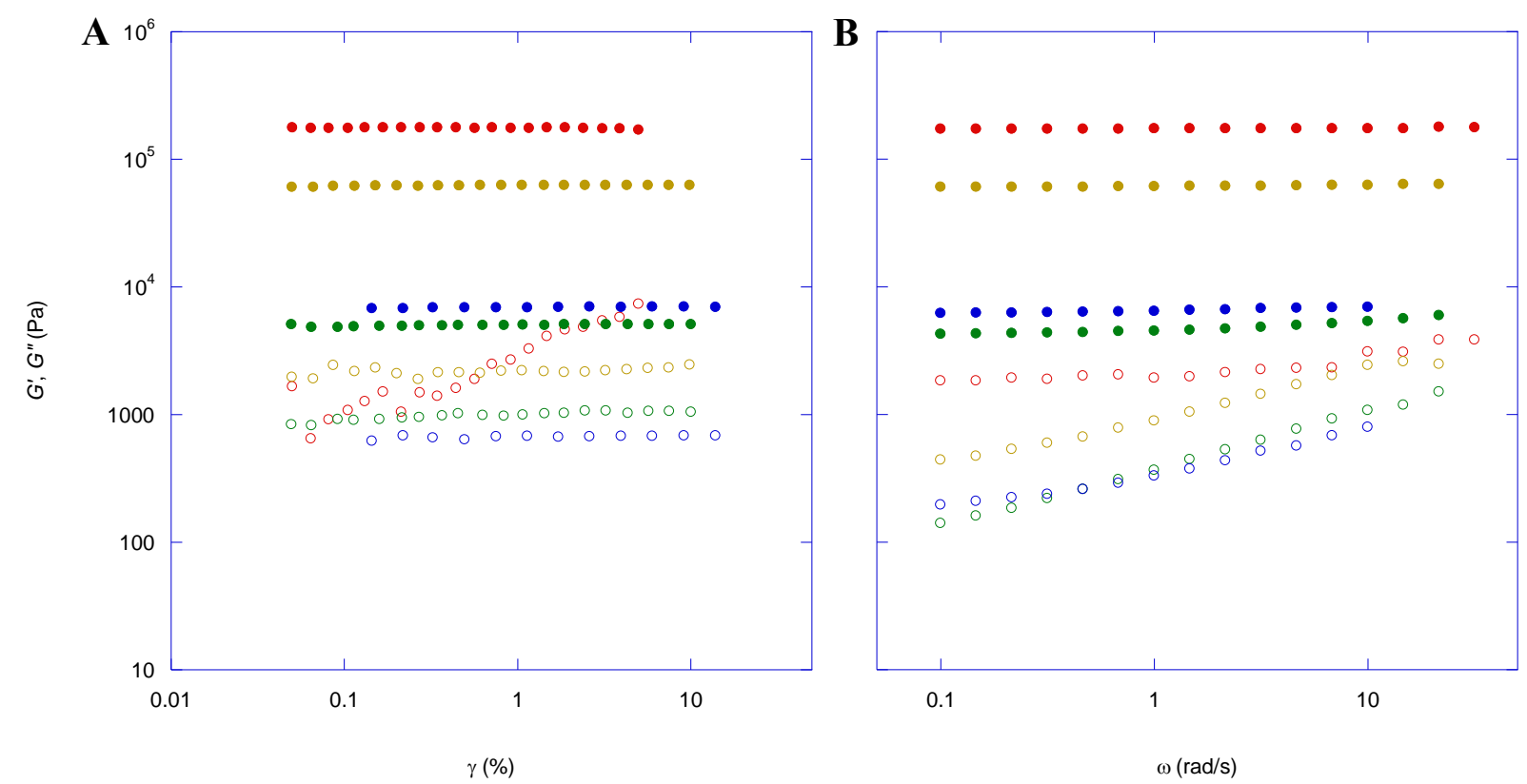

Figure S2. Strain (A) and frequency (B) sweeps of BBNs with molar ratios of MM to XL of 0:1 (red), 10:1 (yellow), 30:1 (green), and 50:1 (blue). $G$ ' and $G$ ” represented by filled and unfilled markers, respectively. Strain sweep performed with angular frequency of $6 \mathrm{rad} / \mathrm{s}$, and frequency sweep performed in the linear viscoelastic region.

Table S3. Shear moduli $(G)$ from CAT and oscillatory shear rheology of BBN elastomers

\begin{tabular}{cccc}
\hline Molar ratio of MM to XL & batch & $\boldsymbol{G}(\mathbf{k P a})^{\mathbf{a}, \mathbf{b}}$ & $\boldsymbol{G}^{\boldsymbol{\prime}}(\mathbf{k P a})^{\mathbf{c}}$ \\
\hline $0: 1$ & 2 & $114 \pm 22$ & 175 \\
$10: 1$ & 2 & $21 \pm 1$ & 62 \\
$30: 1$ & 2 & $2.1 \pm 0.5$ & 5 \\
$50: 1$ & 2 & $1.3 \pm 0.3$ & 7 \\
\hline
\end{tabular}
a. As determined from contact adhesion testing
b. Average value from three trials on one sample with associated standard deviation
c. As determined from frequency sweep at $1 \mathrm{rad} / \mathrm{s}$ 


\section{Characterization of model rough surfaces}
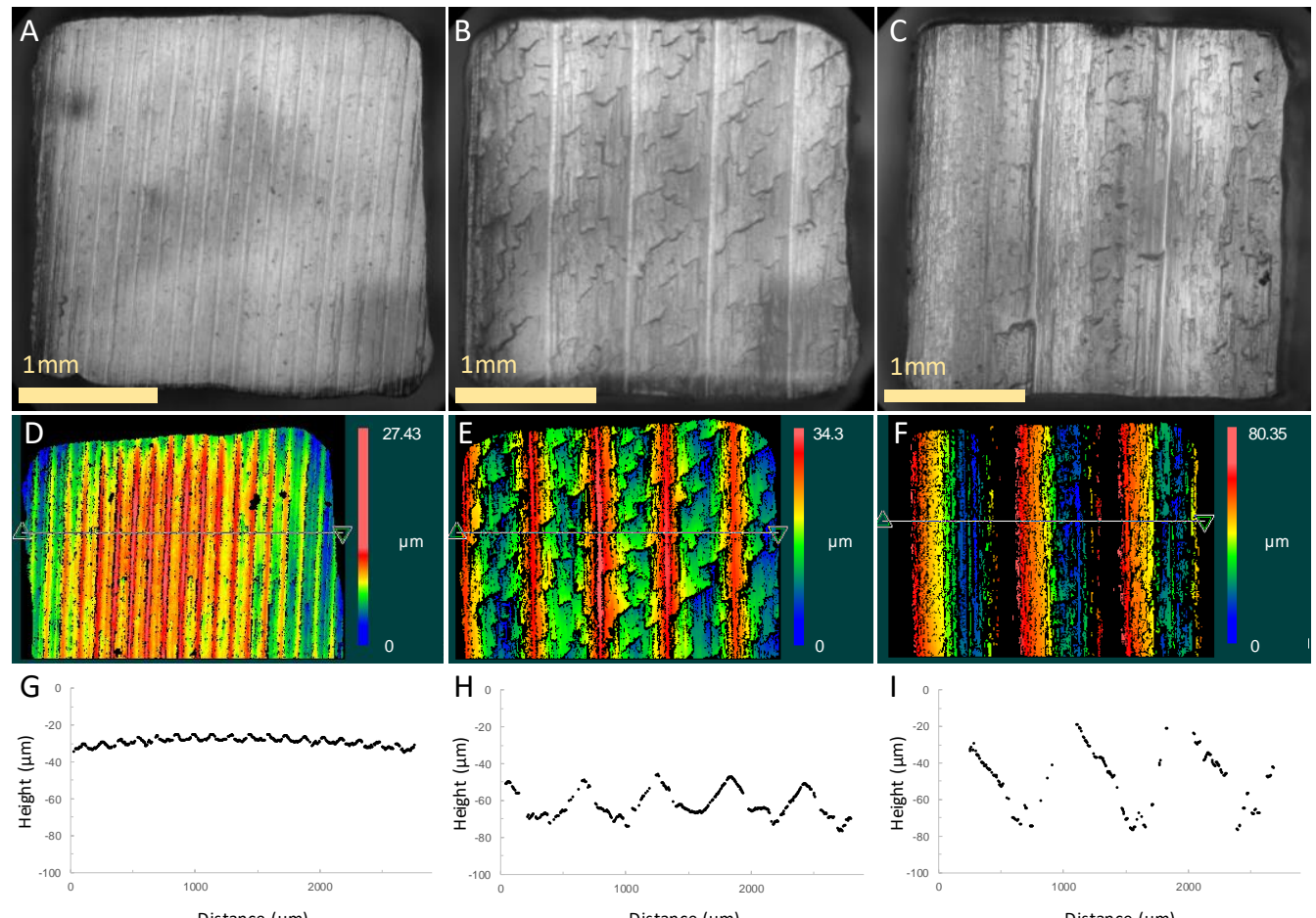

Distance $(\mu \mathrm{m})$

Distance $(\mu \mathrm{m})$

Distance $(\mu \mathrm{m})$

Figure S3. Optical images of the model rough surfaces prepared as replicas of different portions of a microfinish comparator: ST63 (A), ST250 (B), and ST500 (C). Using an optical profilometer, the topographies of the same surfaces were mapped for ST63 (D), ST250 (E), and ST500 (F), with surface profiles of horizonal lines marked on each map plotted for ST63 (G), ST250 (H), and ST500 (I).

Table S4. Roughness values of surface profiles (Figures S3G-I) of the three model rough surfaces.

\begin{tabular}{ccccc}
\hline Rough surface & $\mathbf{P V}(\boldsymbol{\mu \mathbf { m }})^{\mathbf{a}}$ & $\mathbf{R m s}(\boldsymbol{\mu m})$ & $\mathbf{R a}(\boldsymbol{\mu m})$ & $\mathbf{H}_{\mathbf{F}}(\boldsymbol{\mu m})^{\mathbf{b}}$ \\
\hline 63ST & 10 & 2 & 2 & 4 \\
250ST & 31 & 8 & 6 & 23 \\
$500 \mathrm{ST}$ & 59 & 16 & 13 & 55 \\
\hline
\end{tabular}

a. Peak-to-valley height (PV) reported as difference between highest and lowest point along entire substrate length (consisting of multiple v-notch features)

b. Feature height $\left(\mathrm{H}_{\mathrm{F}}\right)$ reported as the difference between highest and lowest point for an individual v-notch feature 


\section{Conformal contact of soft elastomers with rough surfaces}

Methods. Optical images of three model rough surfaces were collected before and during contact with five elastomers of varying $G$. By applying an edge detection image processing protocol to the optical images obtained, certain pixels were mapped as 'edges,' which are pixels that are designated as high contrast and result as black pixels in the edge maps. Image ${ }^{1}$ and a 'Canny Edge Detector' ${ }^{2}$ plug-in were employed to map 'edges' with a Gaussian kernel radius of 0.5, a low threshold of 4.5, and a high threshold of 5.5. A comparison of the edge maps of rough surfaces before and during contact allows for a rough assessment of contact efficiency: a decrease in the number of pixels of high contrast correlates with increased contact efficiency.
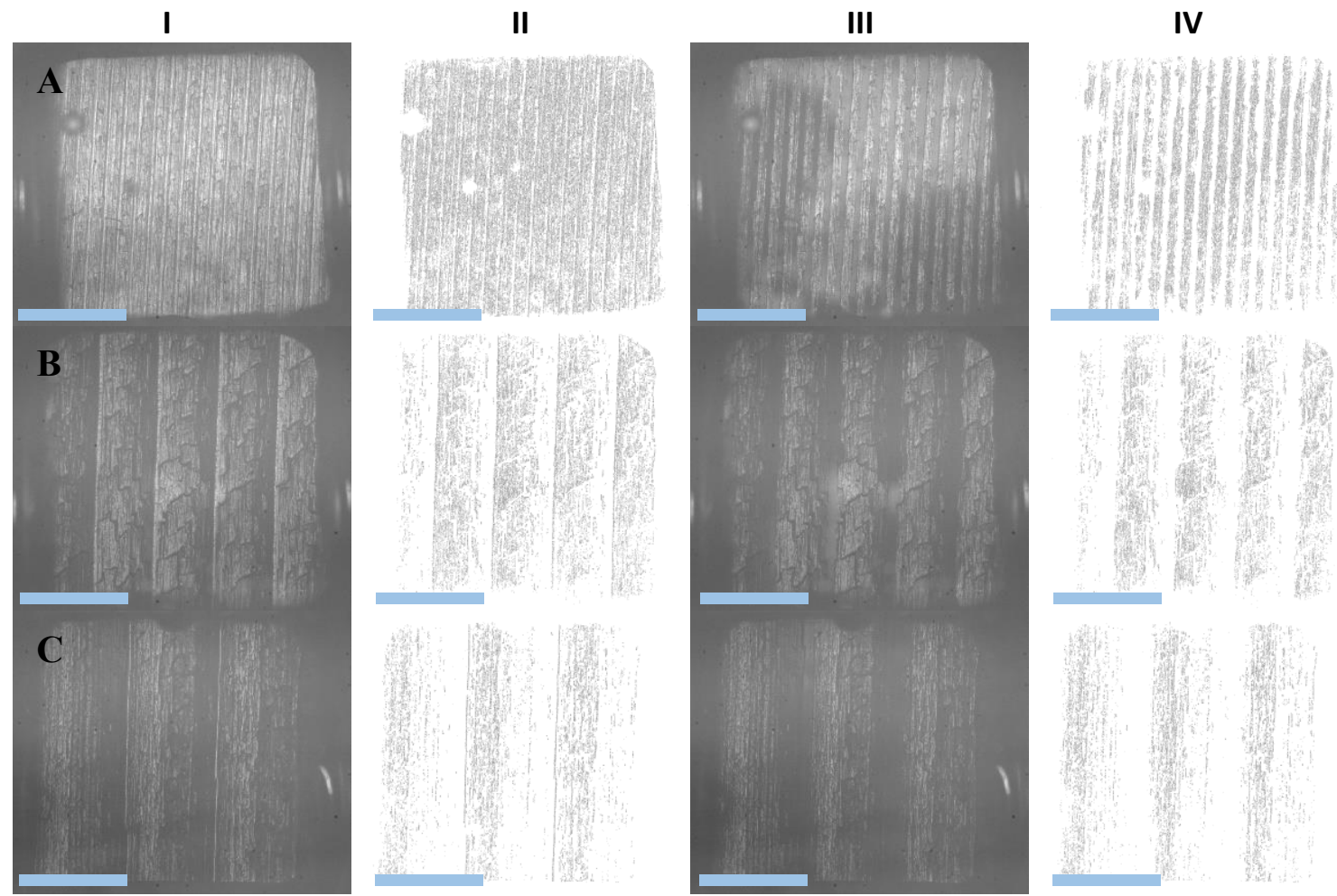

Figure S4. Selected photographs (column I) and corresponding edge maps (column II) before contact and photographs (column III) and corresponding edge maps (column IV) during contact with pressure of $13 \mathrm{mN} / \mathrm{mm}^{2}$ between 10:1 ratio by mass of base to curing agent Sylgard $184(G=352 \mathrm{kPa})$ and model rough surfaces with feature heights of either $4 \mu \mathrm{m}(\mathrm{A}), 30 \mu \mathrm{m}(\mathrm{B})$, or $60 \mu \mathrm{m}$ (C). Scale bar $=1 \mathrm{~mm}$. 

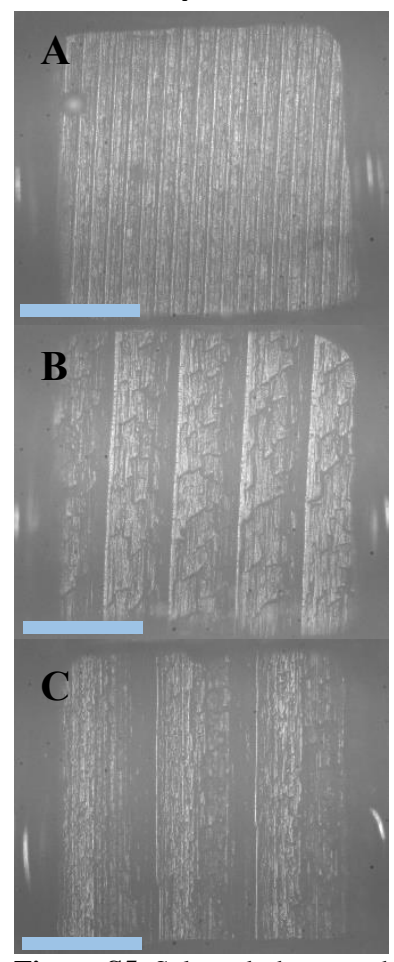

II
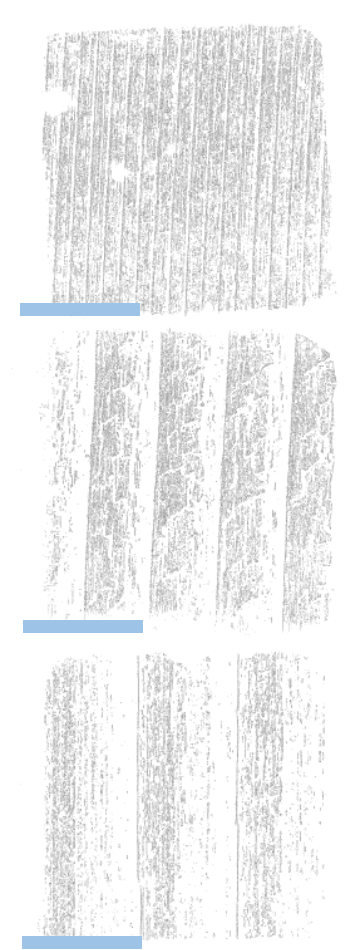

III

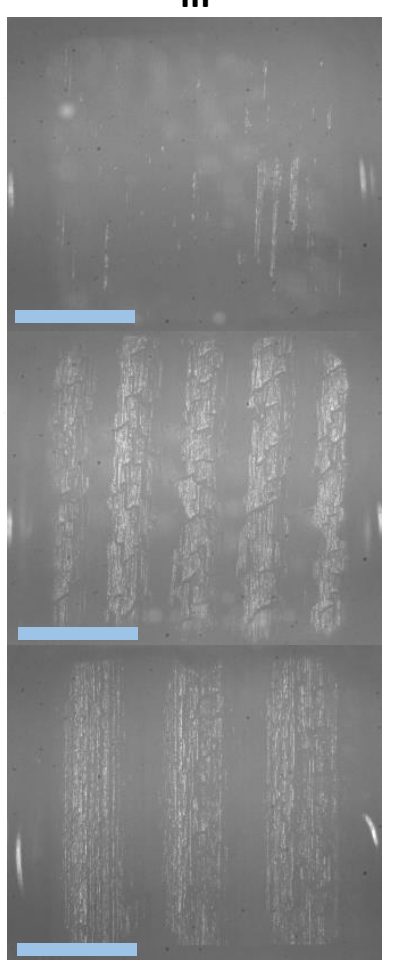

IV

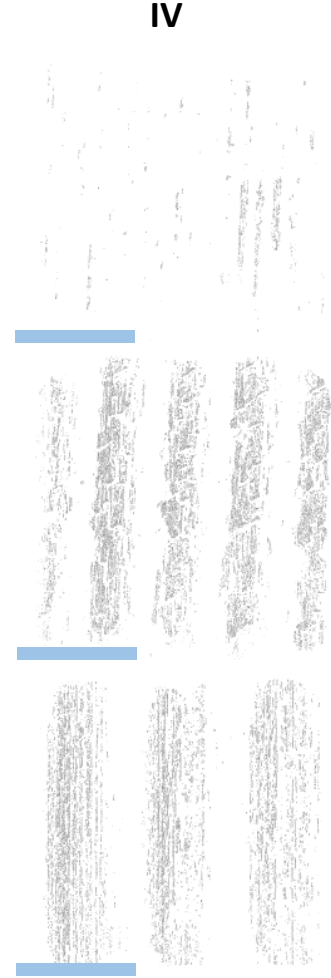

Figure S5. Selected photographs (column I) and corresponding edge maps (column II) before contact and photographs (column III) and corresponding edge maps (column IV) during contact with pressure of $13 \mathrm{mN} / \mathrm{mm}^{2}$ between 20:1 ratio by mass of base to curing agent Sylgard $184(G=126 \mathrm{kPa})$ and model rough surfaces with feature heights of either $4 \mu \mathrm{m}(\mathrm{A}), 30 \mu \mathrm{m}(\mathrm{B})$, or $60 \mu \mathrm{m}$ (C). Scale bar $=1 \mathrm{~mm}$.

I
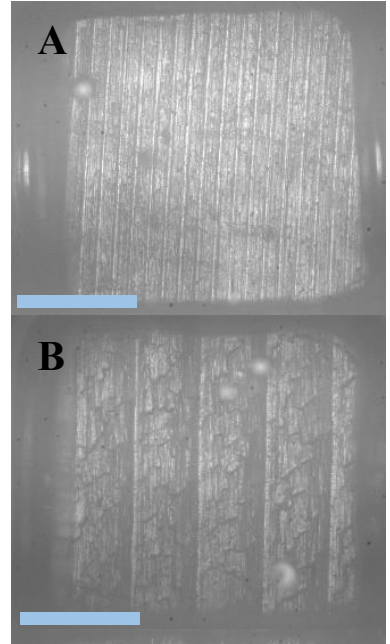

C
II
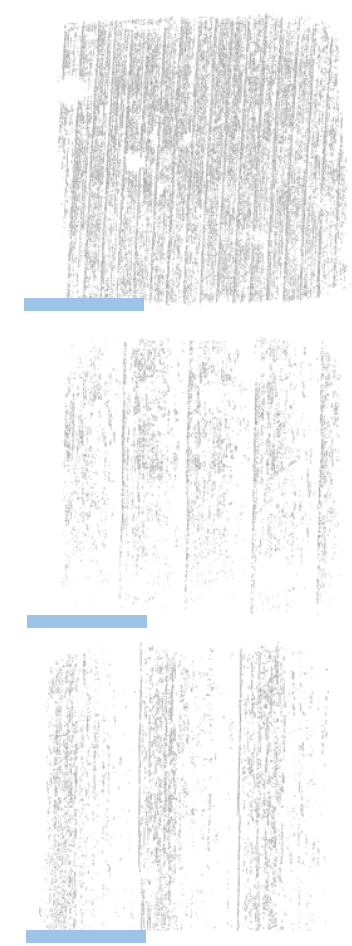

III

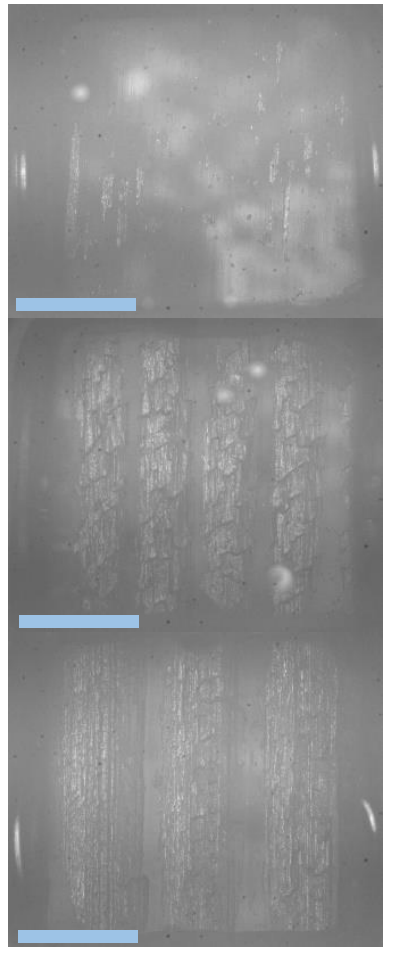

IV

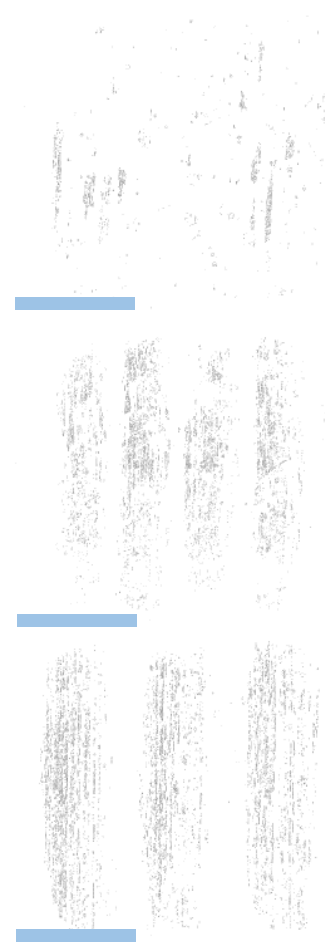

Figure S6. Selected photographs (column I) and corresponding edge maps (column II) before contact and photographs (column III) and corresponding edge maps (column IV) during contact with pressure of $13 \mathrm{mN} / \mathrm{mm}^{2}$ between a BBN with a molar ratio of 0:1 MM to XL $(G=114 \mathrm{kPa})$ and model rough surfaces with feature heights of either $4 \mu \mathrm{m}(\mathrm{A}), 30 \mu \mathrm{m}(\mathrm{B})$, or $60 \mu \mathrm{m}(\mathrm{C})$. Scale bar $=1 \mathrm{~mm}$. 


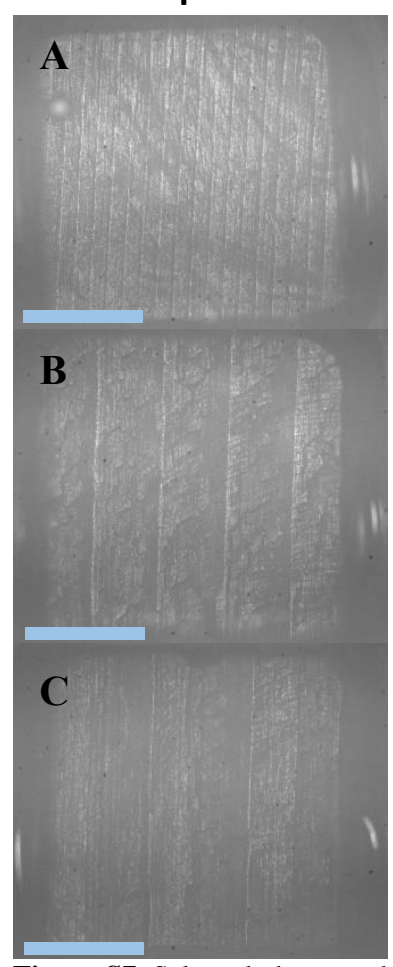

II
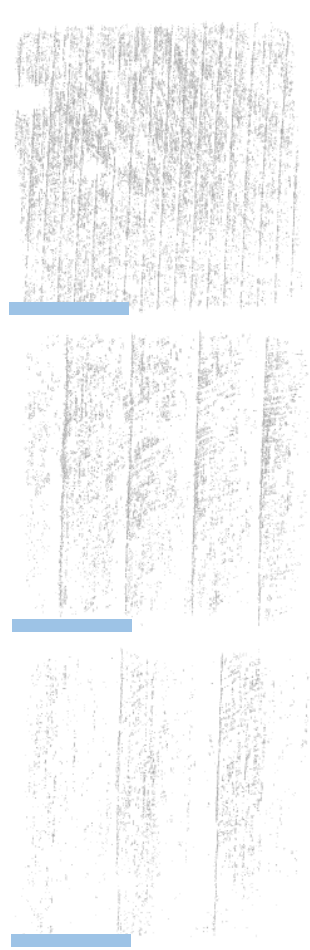

III

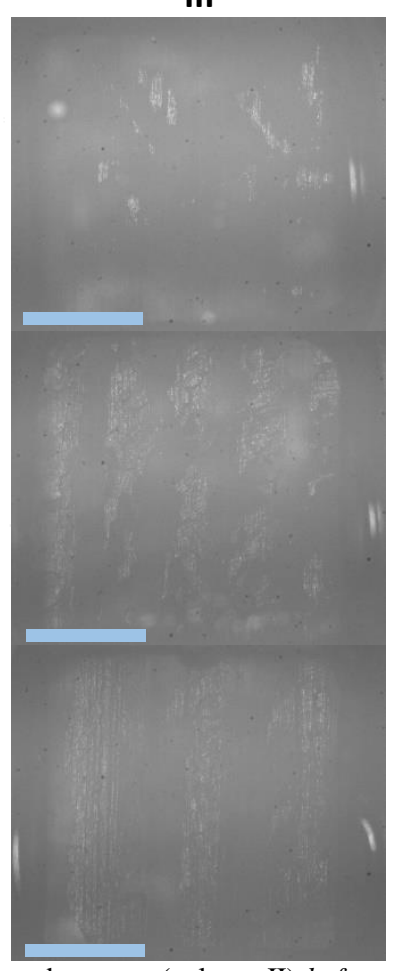

IV

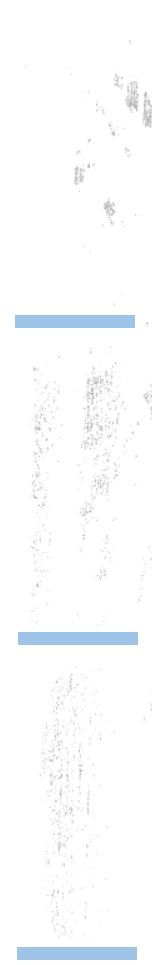

Figure S7. Selected photographs (column I) and corresponding edge maps (column II) before contact and photographs (column III) and corresponding edge maps (column IV) during contact with pressure of $13 \mathrm{mN} / \mathrm{mm}^{2}$ between a BBN with a molar ratio of 10:1 MM to XL $(G=21 \mathrm{kPa})$ and model rough surfaces with feature heights of either $4 \mu \mathrm{m}(\mathrm{A}), 30 \mu \mathrm{m}(\mathrm{B})$, or $60 \mu \mathrm{m}(\mathrm{C})$. Scale bar $=1 \mathrm{~mm}$.

I

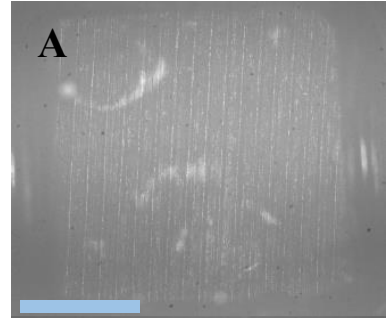

B

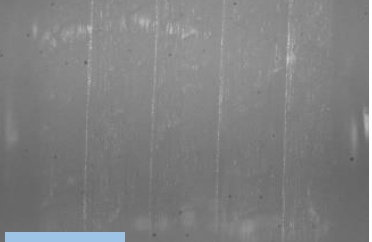

C
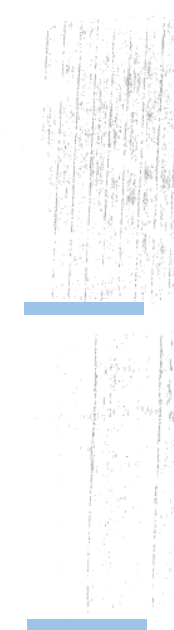

II

III

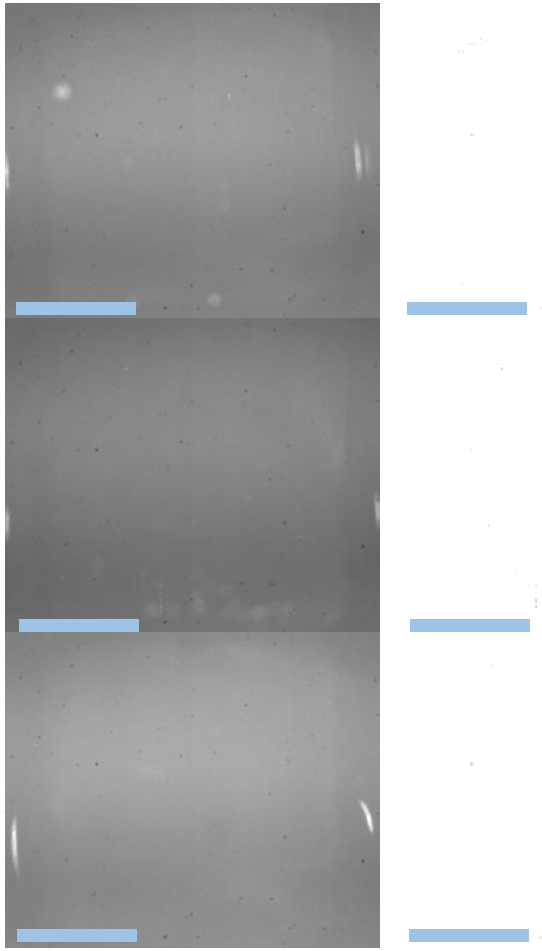

IV

\footnotetext{
Figure S8. Selected photographs (column I) and corresponding edge maps (column II) before contact and photographs (column III) and corresponding edge maps (column IV) during contact with pressure of $13 \mathrm{mN} / \mathrm{mm}^{2}$ between a BBN with a molar ratio of 50:1 MM to XL $(G=1 \mathrm{kPa})$ and model rough surfaces with feature heights of either $4 \mu \mathrm{m}(\mathrm{A}), 30 \mu \mathrm{m}(\mathrm{B})$, or $60 \mu \mathrm{m}(\mathrm{C})$. Scale bar $=1 \mathrm{~mm}$.
} 


\section{Conformal contact of hydrogels with rough surfaces}
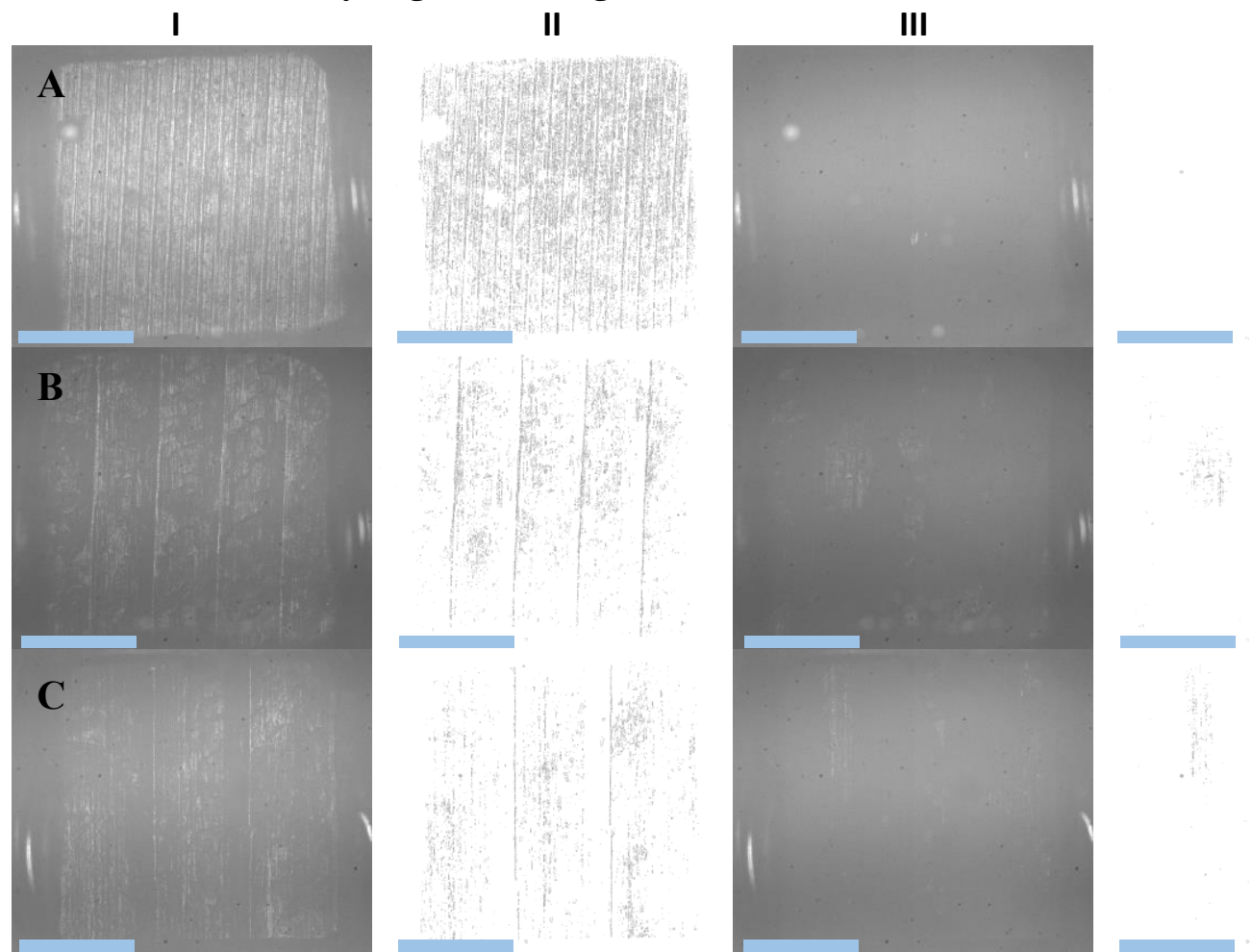

IV

Figure S9. Selected photographs (column I) and corresponding edge maps (column II) before contact and photographs (column III) and corresponding edge maps (column IV) during contact with pressure of $13 \mathrm{mN} / \mathrm{mm}^{2}$ between a 5 mass $\%$ agarose hydrogel $(G=41 \mathrm{kPa})$ and model rough surfaces with feature heights of either $4 \mu \mathrm{m}(\mathrm{A}), 30 \mu \mathrm{m}(\mathrm{B})$, or $60 \mu \mathrm{m}(\mathrm{C})$. Scale bar $=1 \mathrm{~mm}$.

I

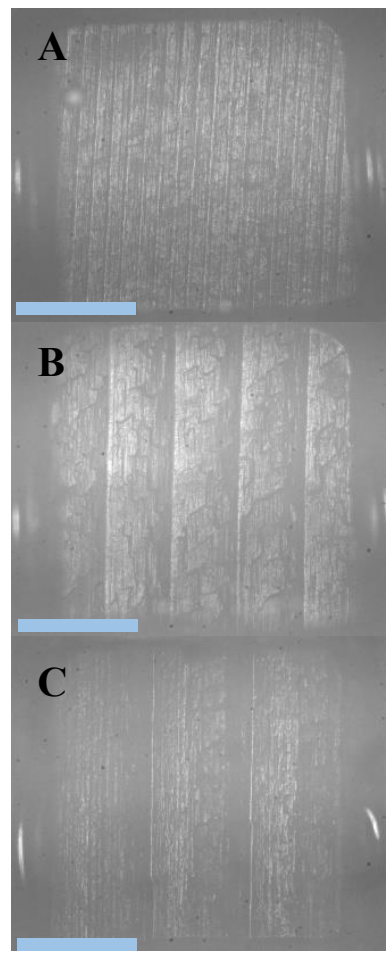

II
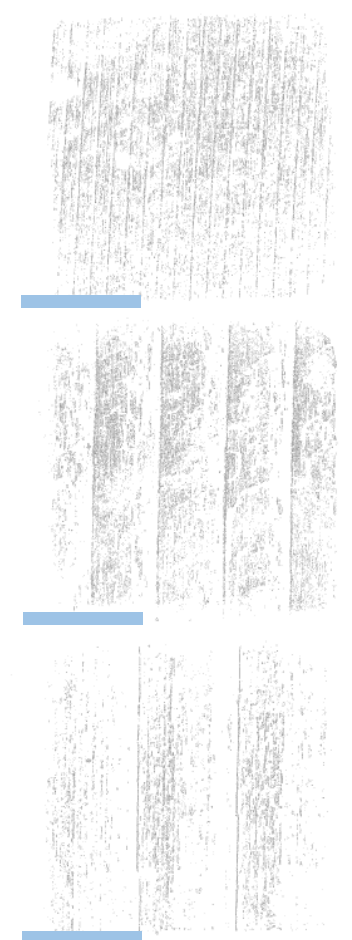

III

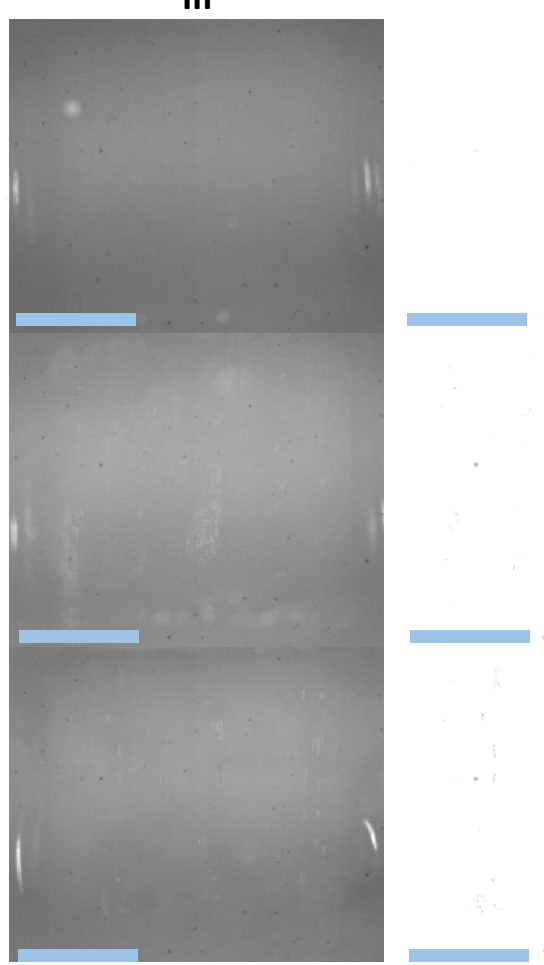

IV

Figure S10. Selected photographs (column I) and corresponding edge maps (column II) before contact and photographs (column III) and corresponding edge maps (column IV) during contact with pressure of $13 \mathrm{mN} / \mathrm{mm}^{2}$ between a 5 mass \% gellan hydrogel $(G=48 \mathrm{kPa})$ and model rough surfaces with feature heights of either $4 \mu \mathrm{m}$ (A), $30 \mu \mathrm{m}$ (B), or $60 \mu \mathrm{m}$ (C). Scale bar $=1 \mathrm{~mm}$. 


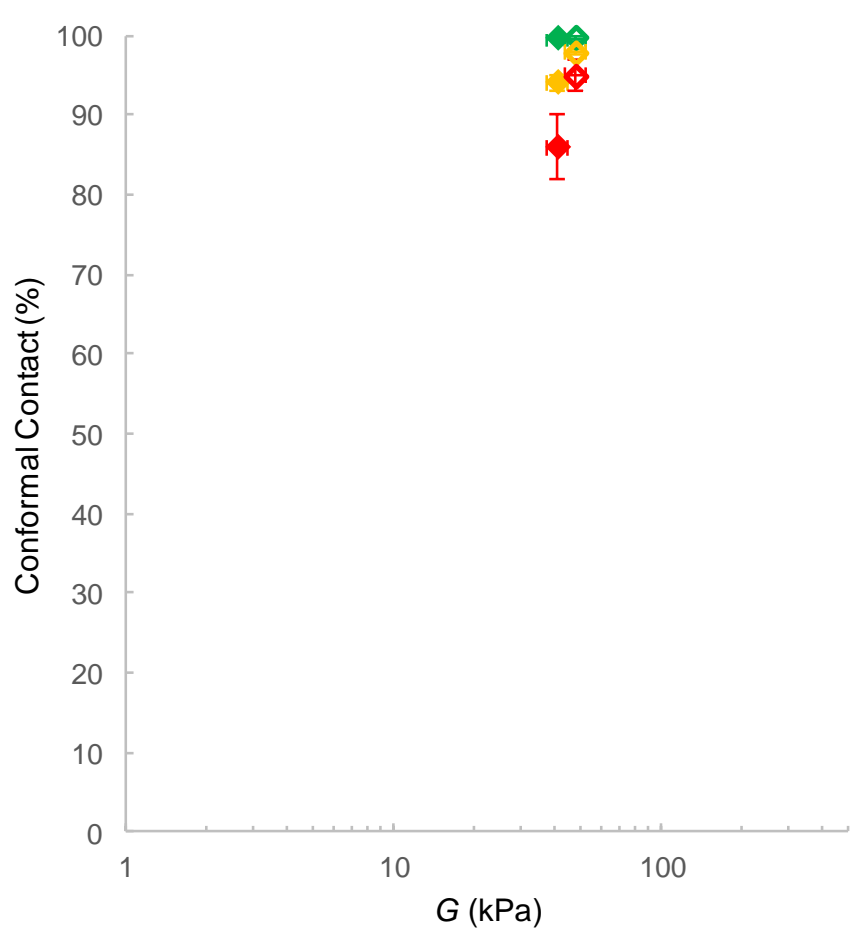

Figure S11. The percent conformal contact for hydrogels (approximately $3 \mathrm{~mm}$ thick) of varying $G$ in contact with three rough surfaces. 5 mass \% agarose (closed diamonds) and gellan (open diamonds) hydrogels were pressed against rough surfaces with feature heights of approximately $4 \mu \mathrm{m}$ (green), $30 \mu \mathrm{m}$ (yellow), and $60 \mu \mathrm{m}$ (red) with an applied pressure of $13 \mathrm{mN} / \mathrm{mm}^{2}$. Error bars designate the standard deviation from three trials of three different contact locations on the same sample.

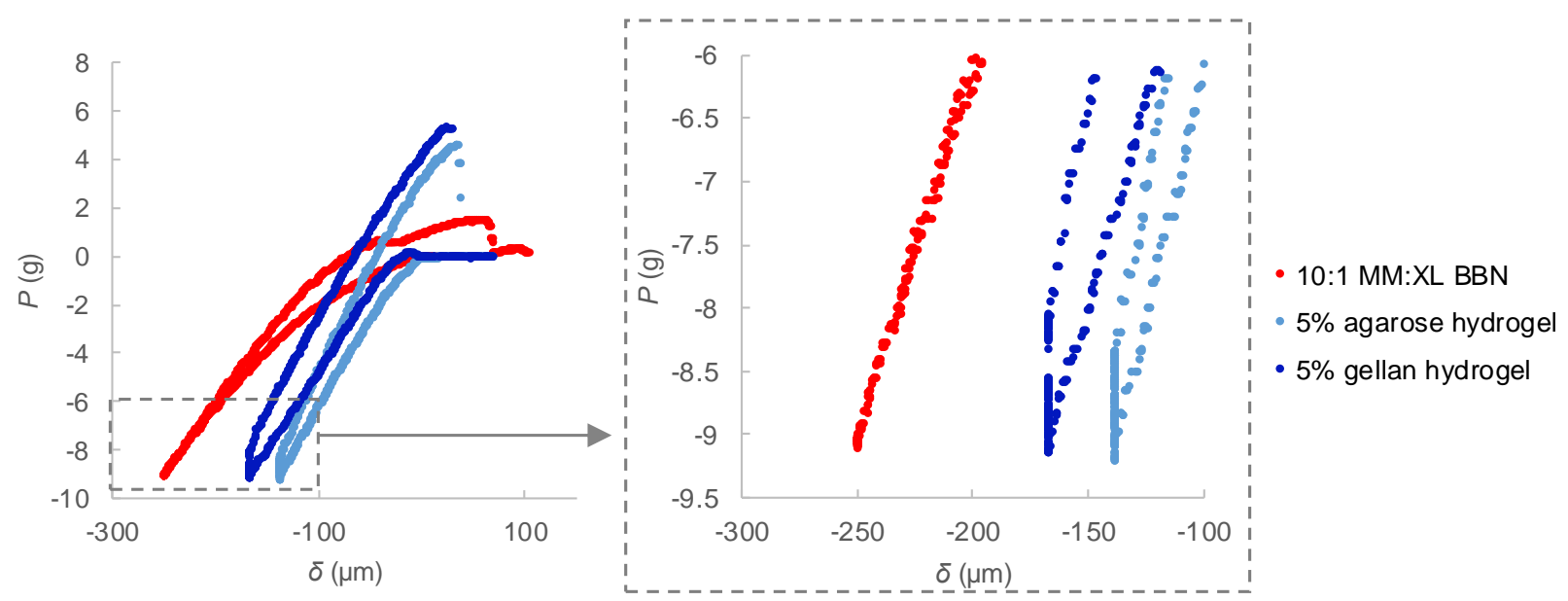

Figure S12. Examples of force-displacement curves with a 30 second dwell at the maximum displacement for the contact experiments of a BBN with a molar ratio of 10:1 MM to XL (red), a 5 mass \% agarose hydrogel (light blue), and a 5 mass \% gellan hydrogel (dark blue). 
Removal of particulates from a soil-coated surface
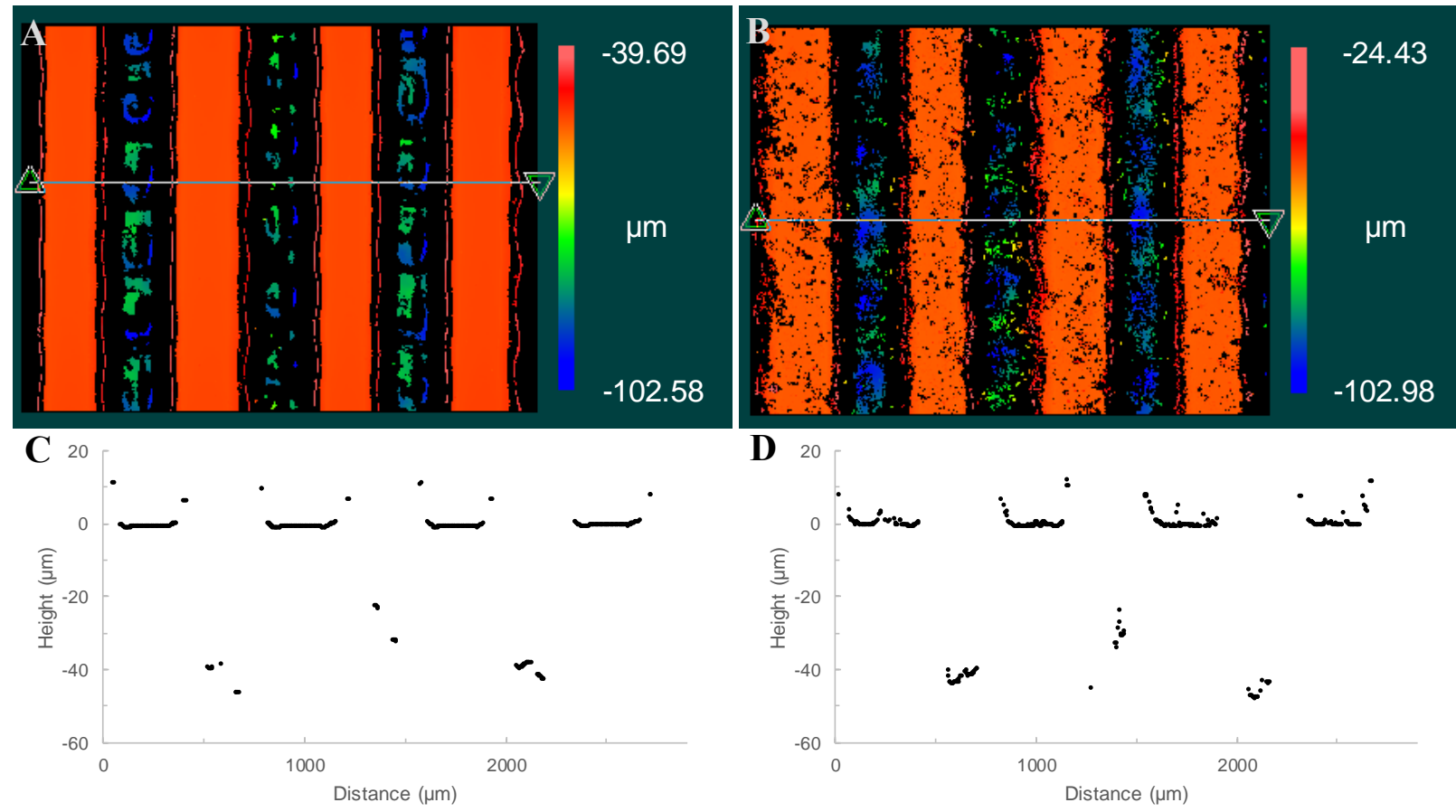

Figure S13. Interferograms of laser-engraved acrylic substrate before (A) and after (B) coating with artificial soil mixture, with surface profiles of horizonal lines marked on each map plotted before (C) and after (D).

Table S5. Roughness values of surface profiles (Figures S13 C,D) of the engraved acrylic surface before and after coating with artificial soil.

\begin{tabular}{cccc}
\hline Acrylic substrate & Peak-Valley $(\boldsymbol{\mu m})$ & Rms $(\boldsymbol{\mu m})$ & Ra $(\boldsymbol{\mu m})$ \\
\hline Before soil application - surface profile & 57.6 & 13.2 & 8.7 \\
After soil application - surface profile & 59.9 & 16.2 & 12.2 \\
\hline
\end{tabular}




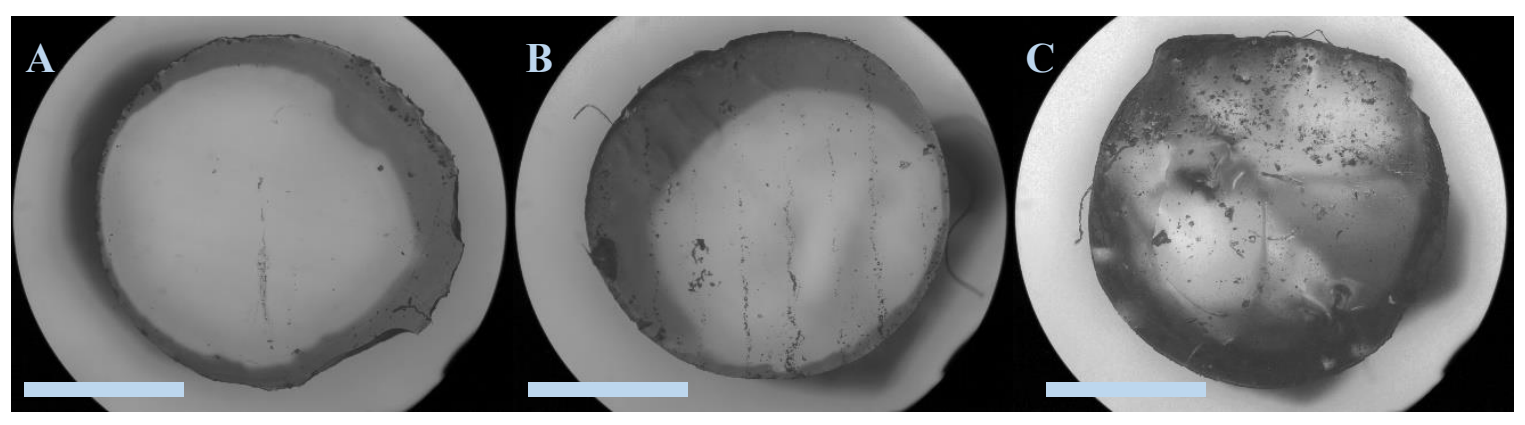

Figure S14. Optical images of elastomers after pressing and peeling from soil-coated, laser-engraved, acrylic substrates: a 10:1 ratio by mass of base to curing agent Sylgard sample (A) of $G=352 \mathrm{kPa}$, a 0:1 molar ratio of MM to XL BBN sample (B) of $G=114 \mathrm{kPa}$, and a molar ratio of 50 to $1 \mathrm{MM}$ to XL BBN sample (C) of $G=1 \mathrm{kPa}$. Scale bar $=1 \mathrm{~mm}$.
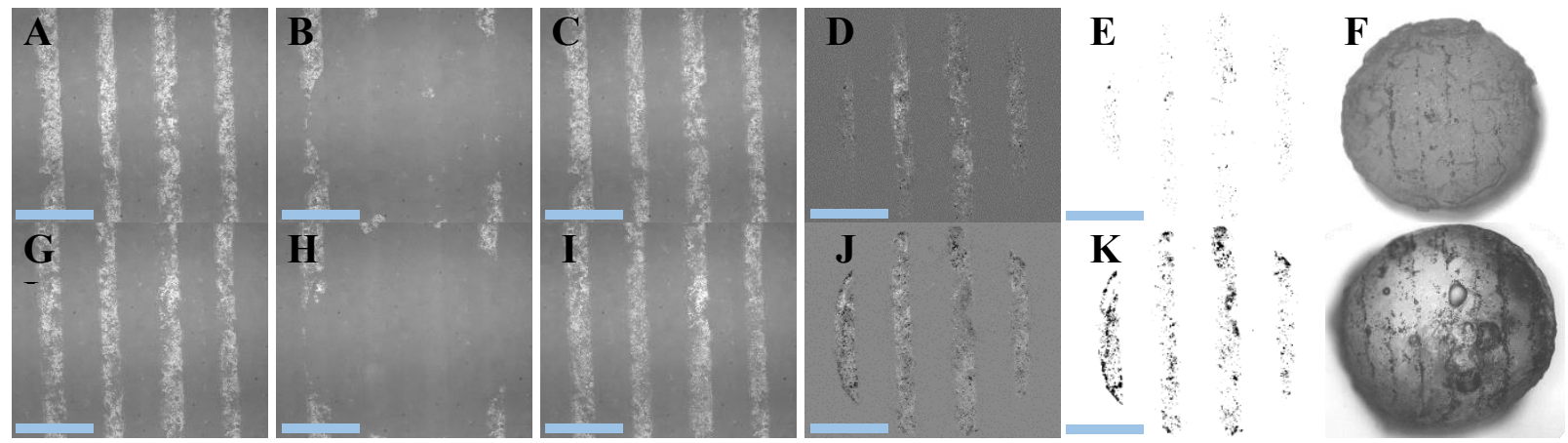

Figure S15. Optical images of soil-coated, engraved acrylic substrate before (A), during (B), and after (C) contact with a 5 mass \% agarose hydrogel $(G=41 \mathrm{kPa})$; and before $(\mathrm{G})$, during $(\mathrm{H})$, and after (I) contact with a 5 mass $\%$ gellan hydrogel $(G=48 \mathrm{kPa})$. Before and after images were subtracted to produce the difference images $(\mathrm{D}, \mathrm{J})$ which were thresholded (E,K) to designate areas cleaned as black and not cleaned as white. Optical images of gels after pressing and peeling from substrates: 5 mass $\%$ agarose hydrogel $(\mathrm{F})$ and 5 mass $\%$ gellan hydrogel $(\mathrm{L})$. Scale bar $=$ $1 \mathrm{~mm}$.

\section{Bibliography}

(1) Schneider, C. A.; Rasband, W. S.; Eliceiri, K. W. NIH Image to ImageJ: 25 Years of Image Analysis. Nat. Methods 2012, 9, 671-675.

(2) Gibara, T. Canny Edge Detector. 2013. 